\title{
Características de los electrolitos de pacientes adultos que acuden por emergencia médica a un hospital general de Lima, Perú
}

\author{
Characteristics of serum electrolytes of adult patients who come by medical emergency to a general \\ hospital in Lima, Peru
}

Javier Antonio Cieza Zevallos 1,a;,b, Christian Bryan Orihuela Jesús ${ }^{\mathrm{c}}$

\section{RESUMEN}

El número de pacientes con desnutrición que demanda servicios de emergencia en hospitales de Lima-MINSA, es considerable y podría relacionarse con alteraciones del medio interno. Tener una idea objetiva cómo esta característica puede ser relevante en esta demanda es importante. Objetivos: Estudiar la magnitud e importancia de las alteraciones electrolíticas en pacientes internados en la emergencia de un hospital nivel III (MINSA Lima-Perú). Material y métodos: Estudio trasversal descriptivo y analítico de los electrolitos en pacientes que acudieron al servicio de emergencia de adultos del Hospital Cayetano Heredia (Lima-Perú) en setiembre del 2016, seleccionado al azar y admitidos para observación mayor de 48 horas. Se consideró el nivel de albúmina sérica, la azoemia y gasometría coexistente al momento del control de los electrolitos. Resultados: La alteración electrolítica más frecuente observada en 206 pacientes fue hipocalcemia (46,7\%); seguida de hiperfosfatemia (32,3\%), hiponatremia $(24,7 \%)$ e hipermagnesemia (18,9\%). La hipoalbuminemia $<3,0 \mathrm{~g} / \mathrm{dl}$ estuvo presente en $50,56 \%$ y $<2,6 \mathrm{~g} / \mathrm{dl}$ en el $31,4 \%$ de los casos. La albuminemia solo mostró correlación significativa con la calcemia $(\mathrm{r}=0,47 ; \mathrm{p}=0,002)$ y la natremia $(r=0,233 ; p=0,028)$. La hiperfosfatemia estuvo relacionada a un modelo dependiente de la creatinina sérica, alcalosis respiratoria y cáncer. La hipomagnesemia e hipermagnesemia se relacionaron con bajos y altos valores de creatinina y urea, respectivamente. Conclusiones: La frecuencia de pacientes con hipoalbuminemia que demandaron servicios de emergencia fue notablemente alta, y su relación más relevante estuvo relacionada con disturbios de la calcemia y la natremia. Otros disturbios relevantes fueron los relativos al magnesio, situación que requiere estudio más exhaustivo.

PALABRAS CLAVE: Atención ambulatoria, adulto, electrólitos, hipoalbuminemia. (Fuente: DeCS BIREME).

\section{SUMMARY}

Patients with malnutrition who demand emergency services in Lima-MINSA hospitals, is considerable and could be related to alterations of the internal medium. Having an objective idea how this feature can be relevant in this patients demand is important. Objectives: To study the magnitude and importance of electrolyte alterations in hospitalized patients in the emergency of a level III hospital (MINSA Lima-Perú). Methods: Descriptive and analytical crosssectional study of electrolytes in patients who attended the emergency service of adults of the Hospital Nacional Cayetano Heredia (Lima-Perú) in September 2016, selected by chance and admitted for observation $>48$ hours. The level of serum albumin, azotemia and coexisting gasometry at the time of electrolyte control was considered. Results: The highest frequency of electrolyte alteration observed in 206 patients was hypocalcemia (46.7\%); followed by hyperphosphatemia (32.3\%), hyponatremia (24.7\%) and hypermagnesemia (18.9\%). Hypoalbuminemia $<3.0 \mathrm{~g} /$ dl was present in $50.56 \%$ and $<2.6 \mathrm{~g} / \mathrm{dl}$ in $31.4 \%$. Albuminemia only had a significant correlation with calcemia

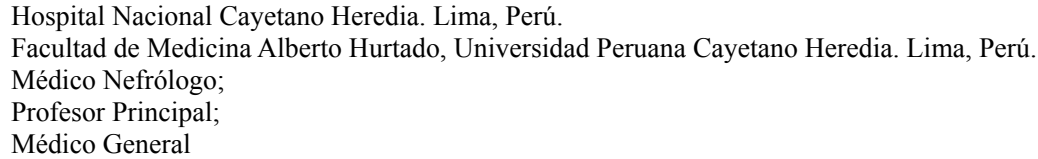


$(\mathrm{r}=0.47 ; \mathrm{p}=0.002)$ and natremia $(\mathrm{r}=0.233 ; \mathrm{p}=0.028)$. Hyperphosphatemia was related to a model dependent on serum creatinine, respiratory alkalosis and cancer. Both, hypomagnesemia and hypermagnesemia were related with low and high creatinine and urea values respectively. Conclusions: The frequency of patients with hypoalbuminemia who demanded emergency services was remarkably high, and their most relevant relationship was related to calcemia and natremia disturbances. Other relevant disturbances were related to magnesium, a situation that requires more exhaustive study.

KEYWORDS: Ambulatory care, adult, electrolytes, hipoalbuminemia. (Source: MeSH BIREME).

\section{INTRODUCCIÓN}

La carga de enfermedad con la cual llega un paciente al servicio de emergencia de un hospital general en Lima Metropolitana usualmente es subestimada porque en la mayoría de las situaciones, acapara la atención del médico el problema agudo que aqueja al paciente, que por lo general distrae al facultativo de los problemas crónicos de larga data, que arrastra el enfermo y que son los que influenciarán en su evolución.

De otro lado, en nuestro país se observa un aumento significativo en la edad de personas que solicitan servicios de emergencia en hospitales terciarios y que requieren internamiento (1). Es sabido que el envejecimiento es un proceso fisiológico multifactorial, donde la capacidad funcional se reduce progresivamente, proceso que se inicia aproximadamente a los 45 años en países en vías de desarrollo y cerca de los 58 años para países desarrollados (2).

En este escenario, la pérdida de esta reserva funcional se acompaña del aumento de desórdenes fisiológicos crónicos en los pacientes. Uno de los más importantes procesos que actúa como mecanismo compensatorio ante esta pérdida funcional progresiva, es la regulación del medio interno. Este proceso permite mantener los compartimentos líquidos corporales de nuestro organismo, dentro de márgenes adecuados para nuestra vida (3). Desórdenes del medio interno frecuentemente son consecuencia de deficiencias nutricionales, donde la albúmina sérica juega un rol importante como marcador nutricional y como regulador de la presión oncótica del líquido intravascular, variable relevante para su idónea funcionalidad (3).

En el Perú, la Dirección Ejecutiva de Vigilancia Alimentaria y Nutricional (DEVAN), informó que entre los años 2012 y 2013 , el $17,7 \%$ de los peruanos adultos presentaron desnutrición crónica y el 44,67\% tuvieron sobrepeso (4). Es pues esperable que los niveles plasmáticos de albúmina se encuentren con frecuencia alterados y asociados con dichas poblaciones, condicionando alteraciones en el medio interno.

La hipoalbuminemia, mayormente evidente en población desnutrida moderada o severa, disminuye la presión oncótica del plasma, produciendo desbalance entre los medios intravascular e intersticial. Al existir menor presión oncótica en el agua plasmática, el líquido se transfiere al agua intersticial generando una "hipovolemia" relativa. Para compensar esta situación, los niveles de aldosterona y de la hormona antidiurética $(\mathrm{ADH})$ se incrementan generando un balance positivo de agua y sodio cuya consecuencia posterior es la hemodilución (3). Esta hipoalbuminemia también genera alteraciones del equilibrio ácido-base, porque al poseer la albúmina carga negativa, actúa como un ácido débil no volátil y por ello la hipoalbuminemia incrementa los niveles de álcalis (principalmente bicarbonato) y se asocia a un alto riesgo de muerte (5).

Es también importante resaltar que numerosos otros factores condicionan carga de enfermedad en las personas; según el estudio TORNASOL son la obesidad, diabetes, hipertensión arterial, desnutrición y dislipidemias (6). Dichos factores predisponen a un deterioro acelerado del medio interno y acortan el tiempo hacia fases avanzadas de enfermedad y daño de órgano blanco.

Por lo enunciado, es importante tener una aproximación integral de los desórdenes electrolíticos de una población potencialmente vulnerable que acude a las emergencias de hospitales de nivel terciario en nuestra sociedad.

Este trabajo tuvo como objetivo estudiar la magnitud y la relevancia de las alteraciones del medio interno de pacientes internados en la emergencia de un hospital nivel III del Ministerio de Salud (MINSA) en Lima, Perú.

\section{MATERIAL Y MÉTODOS}

Estudio trasversal de sondeo, descriptivo y analítico de las principales variables del medio interno de pacientes que acudieron al servicio de emergencia 
de adultos del Hospital Cayetano Heredia en Lima, Perú, en el mes setiembre del año 2016, seleccionados al azar y que fueron admitidos para observación mayor de 48 horas.

Se recopiló de la historia clínica la información de los valores séricos de electrolitos, hemograma, gasometría, urea, creatinina, peso, edad y sexo, al momento de ingreso. La recopilación de la información y realización del estudio fue aprobada por el Comité de Ética del hospital.

Se calculó un tamaño de muestra en función de un promedio mensual de admisiones de 442 pacientes/ mes, cuya probabilidad teórica de tener una alteración del medio interno fue $50 \%$, un margen de error del $5 \%$ y una confianza del $95 \%$. El resultado determinó la necesidad de tener una muestra mínima de 206 observaciones.

El muestreo fue accidental hasta alcanzar el tamaño de muestra requerida en el mes seleccionado y de no conseguirlo se prosiguió en el mes subsiguiente. Se incluyeron pacientes de 18 años o más y valores de creatinina, sodio, potasio y cloro séricos.

En ellos se registró además el sexo, peso, estatura, con lo que se calculó su índice de masa corporal (IMC). Se definido desnutrido IMC $<19$, normal IMC entre 19 y 24 , sobrepeso IMC entre 25 y 29 y obesidad $I M C \geq 30$. Los electrolitos, fueron registrados como su valor numérico informado por el laboratorio y luego fueron estratificados para efectos de análisis de contingencias. La ficha de información también consideró la existencia o no de sistemas disfuncionales en cada paciente al momento de la admisión. El análisis estadístico en una primera fase fue descriptivo de las variables sodio, potasio, calcio y magnesio. En una segunda etapa se estudió la existencia de correlación entre estas variables y la albúmina sérica y posteriormente, se analizó mediante análisis de regresión logística multinomial las variables electrolíticas considerando cada una de ellas como variable dependiente y la existencia o ausencia de diversas alteraciones en su gasometría arterial, existencia de sistemas comprometidos y grado de alteración de la albúmina sérica como variables asociadas.

Se consideró como valores normales, sodio $\geq 135$ y $\leq 144 \mathrm{mEq} / 1$; potasio $\geq 3,5 \mathrm{y} \leq 5,49 \mathrm{mEq} / 1 ;$ cloro $\geq 96 \mathrm{y}$ $\leq 110 \mathrm{mEq} / \mathrm{l}$; calcio $\geq 8,5 \mathrm{y} \leq 10,5 \mathrm{mg} / \mathrm{dl}$; fósforo $\geq 2,5 \mathrm{y}$ $\leq 4,5 \mathrm{mg} / \mathrm{dl}$, y magnesio $\geq 1,7 \mathrm{y} \leq 2,2 \mathrm{mg} / \mathrm{dl}$.

Para la albúmina sérica se consideraron tres categorías: una inferior que incluyó pacientes con albúmina $\leq 2,5 \mathrm{~g} / \mathrm{dl}$, una intermedia con valores entre 2,6 y $3,0 \mathrm{~g} / \mathrm{dl}$ y una tercera que incluyó pacientes con albúmina $\geq 3,0 \mathrm{~g} / \mathrm{dl}$.

Respecto a la gasometría arterial se consideró si el paciente tuvo o no acidosis metabólica, acidosis respiratoria, alcalosis metabólica, alcalosis respiratoria y un trastorno mixto de acidosis metabólica con alcalosis respiratoria.

Respecto al cuadro clínico que aquejaba el paciente, se consideró si estaba o no en estado de sepsis, disfunción cardíaca, respiratoria o neurológica. La disfunción renal no fue considerada como criterio porque se analizaron la urea y creatinina como variables continuas: la creatinina fue considerada baja si fue $<0,5 \mathrm{mg} / \mathrm{dl}$, normal si estuvo entre 0,5 y $1,1 \mathrm{mg} /$ $\mathrm{dl}$, y elevada si el valor fue $>1,1 \mathrm{mg} / \mathrm{dl}$. La urea fue considerada en cuatro categorías: $<20,0 \mathrm{mg} / \mathrm{dl}$; entre 20 y $40 \mathrm{mg} / \mathrm{dl}$; entre 41 y $79 \mathrm{mg} / \mathrm{dl}$ y $\geq 80 \mathrm{mg} / \mathrm{dl}$.

Para el análisis se utilizó el paquete estadístico SPSS v 18 y se consideró estadísticamente significativo $\mathrm{p}<0,05$.

\section{RESULTADOS}

Se incluyeron 221 resultados de pacientes $(48,2 \%$ mujeres y $51,8 \%$ varones). La edad media fue $54,8 \pm$ 10 años. La edad no fue diferente en relación al sexo.

Tabla 1. Valor medio, máximo, mínimo y proporción de valores observados dentro de lo normal, inferior y superior a lo normal.

\begin{tabular}{lcccccc}
\hline Electrolito & Media \pm DS & Min-Max & $\begin{array}{c}\text { Normales } \\
(\%)\end{array}$ & $\begin{array}{c}<\text { Normal } \\
(\%)\end{array}$ & $\begin{array}{c}>\text { Normal } \\
(\%)\end{array}$ & Total \\
\hline Sodio $\mathrm{mEq} / \mathrm{l}$ & $139,1 \pm 6,5$ & $117-167$ & $138(63,0)$ & $54(24,7)$ & $27(12,3)$ & 219 \\
Potasio $\mathrm{mEq} / 1$ & $4,27 \pm 0,75$ & $2,8-7,38$ & $177(80,8)$ & $25(11,4)$ & $17(7,8)$ & 219 \\
Cloro $\mathrm{mEq} / \mathrm{l}$ & $100,3 \pm 6,5$ & $81-128$ & $161(73,5)$ & $47(21,5)$ & $11(5,0)$ & 219 \\
Calcio $\mathrm{mg} / \mathrm{dl}$ & $8,41 \pm 1,01$ & $4,8-11,9$ & $47(52,2)$ & $42(46,7)$ & $1(1,1)$ & 90 \\
Magnesio $\mathrm{mg} / \mathrm{dl}$ & $2,06 \pm 0,59$ & $1,1-4,9$ & $69(65,1)$ & $17(16,0)$ & $20(18,9)$ & 106 \\
Fósforo $\mathrm{mg} / \mathrm{dl}$ & $4,45 \pm 1,67$ & $1,5-10,2$ & $61(63,5)$ & $4(4,2)$ & $31(32,3)$ & 96 \\
\hline
\end{tabular}


Los valores de los electrolitos, así como la frecuencia de valores hallados dentro de lo normal, encima de lo normal y debajo de lo normal se muestran en la tabla 1.

El análisis bivariado de correlación entre los electrolitos (que incluyó a la albúmina sérica), mostró que el sodio correlacionó significativamente con el cloro y el fósforo $(\mathrm{p}=0,000)$, el calcio y la albúmina $(\mathrm{p}<0,05)$. El potasio solamente correlacionó significativamente con el fósforo $(\mathrm{p}=0,002)$. El fósforo correlacionó fuertemente con el sodio, potasio y cloro, y en menor medida con el calcio $(\mathrm{p}=0,019)$; no habiendo correlación significativa con el magnesio ni con la albúmina. El calcio correlacionó fuertemente con la albúmina $(\mathrm{p}=0,002)$ y con el cloro $(\mathrm{p}=0,003)$, $\mathrm{y}$ en menor magnitud con el sodio y el fósforo, no mostrando correlación con el magnesio ni con el potasio. El magnesio no mostró correlación con los electrolitos estudiados ni con los niveles de albúmina sérica (Tabla 2).

En el análisis de regresión logística multinomial de los electrolitos, el sodio solo mostró asociación con la albuminemia (Tabla 3), mientras que el potasio no mostró asociación estadísticamente significativa con

Tabla 2. Correlación simple de los diferentes electrolitos medidos en sangre y de la albúmina sérica entre sí.

\begin{tabular}{|c|c|c|c|c|c|c|c|}
\hline & & $\mathbf{K}^{+}$ & $\mathrm{Cl}^{-}$ & $\mathbf{M g}^{++}$ & $\mathbf{P}^{++}$ & $\mathbf{C a T}^{++}$ & $\begin{array}{l}\text { Albúmina } \\
(\mathrm{g} / \mathrm{dl})\end{array}$ \\
\hline \multirow{3}{*}{$\mathrm{Na}^{+}$} & Correlación de Pearson & $-0,079$ & $0,762^{* *}$ & 0,017 & $-0,350^{* *}$ & $0,252^{*}$ & $0,233^{*}$ \\
\hline & Sig. (bilateral) & 0,242 & 0,000 & 0,864 & 0,000 & 0,016 & 0,028 \\
\hline & $\mathrm{n}$ & 219 & 219 & 106 & 96 & 90 & 89 \\
\hline \multirow{3}{*}{$\mathrm{K}^{+}$} & Correlación de Pearson & & 0,055 & 0,190 & $0,310^{* *}$ & 0,204 & 0,055 \\
\hline & Sig. (bilateral) & & 0,420 & 0,051 & 0,002 & 0,053 & 0,610 \\
\hline & $\mathrm{n}$ & & 219 & 106 & 96 & 90 & 89 \\
\hline \multirow{3}{*}{$\mathrm{Cl}^{-}$} & Correlación de Pearson & & & 0,015 & $-0,345^{* *}$ & $0,307^{* *}$ & 0,130 \\
\hline & Sig. (bilateral) & & & 0,875 & 0,001 & 0,003 & 0,226 \\
\hline & $\mathrm{n}$ & & & 106 & 96 & 90 & 89 \\
\hline \multirow{3}{*}{$\mathrm{Mg}^{++}$} & Correlación de Pearson & & & & 0,168 & 0,101 & 0,272 \\
\hline & Sig. (bilateral) & & & & 0,127 & 0,400 & 0,056 \\
\hline & $\mathrm{n}$ & & & & 84 & 72 & 50 \\
\hline \multirow{3}{*}{$\mathrm{P}^{++}$} & Correlación de Pearson & & & & & $-0,281^{*}$ & 0,088 \\
\hline & Sig. (bilateral) & & & & & 0,019 & 0,555 \\
\hline & $\mathrm{n}$ & & & & & 70 & 47 \\
\hline \multirow{3}{*}{$\mathrm{Ca}^{++}$} & Correlación de Pearson & & & & & & $0,470^{* *}$ \\
\hline & Sig. (bilateral) & & & & & & 0,002 \\
\hline & $\mathrm{n}$ & & & & & & 42 \\
\hline
\end{tabular}

** La correlación es significativa al nivel 0,01 (bilateral). * La correlación es significante al nivel 0,05 (bilateral).

Tabla 3. Asociación entre la natremia y la albuminemia en pacientes atendidos en la emergencia.

\begin{tabular}{lcccccccc}
\hline & \multicolumn{4}{c}{ ALBÚMINA SÉRICA } & \multicolumn{2}{c}{ TOTAL } \\
\cline { 2 - 7 } & \multicolumn{2}{c}{$<\mathbf{2 , 6}$ g/d } & \multicolumn{2}{c}{$\mathbf{2 , 6 - 2 , 9}$ g/dl } & \multicolumn{2}{c}{$>\mathbf{3 , 0}$ g/dl } & & \\
\hline Hiponatremia & 11 & $39,29 \%$ & 4 & $23,53 \%$ & 6 & $13,64 \%$ & 21 & $23,60 \%$ \\
Normonatemia & 15 & $53,57 \%$ & 8 & $47,06 \%$ & 34 & $77,27 \%$ & 57 & $64,04 \%$ \\
Hipernatremia & 2 & $7,14 \%$ & 5 & $29,41 \%$ & 4 & $9,09 \%$ & 11 & $12,36 \%$ \\
TOTAL & 28 & & 17 & & 44 & & 89 & \\
\hline
\end{tabular}

$p=0,016$ 
Tabla 4. Asociación entre calcemia y la albuminemia de pacientes atendidos en la emergencia.

\begin{tabular}{lcccccccc}
\hline & \multicolumn{9}{c}{ ALBÚMINA SÉRICA } & \multicolumn{2}{c}{ TOTAL } \\
\cline { 2 - 7 } & \multicolumn{2}{c}{$<\mathbf{2 , 6}$ g/dl } & $\mathbf{2 , 6 - 2 , 9}$ g/dl & \multicolumn{2}{c}{$>\mathbf{3 , 0}$ g/dl } & & \\
\hline Hipocalccemia & 12 & $80,0 \%$ & 3 & $33,3 \%$ & 5 & $27,8 \%$ & 20 & $47,6 \%$ \\
Normocalcemia & 3 & $20,0 \%$ & 6 & $66,7 \%$ & 12 & $66,7 \%$ & 21 & $50,0 \%$ \\
Hipercalcemia & 0 & $0,0 \%$ & 0 & $0,0 \%$ & 1 & $5,6 \%$ & 1 & $2,4 \%$ \\
TOTAL & 15 & & 9 & & 18 & & 42 & \\
\hline
\end{tabular}

$p=0,03$

Tabla 5. Modelo final de regresión logística multinomial relacionado al fósforo sérico de pacientes atendidos en la emergencia.

\begin{tabular}{|c|c|c|c|}
\hline & \multirow{2}{*}{$\begin{array}{c}\text { Criterio de ajuste del } \\
\text { modelo } \\
-2 \text { log verosimilitud del } \\
\text { modelo reducido }\end{array}$} & \multicolumn{2}{|c|}{$\begin{array}{c}\text { Contrastes de la razón de } \\
\text { verosimilitud }\end{array}$} \\
\hline & & Chi-cuadrado & $p$ \\
\hline Intersección & $19,872^{\mathrm{a}}$ & 0,000 & 0,000 \\
\hline Creatinina sérica & 33,486 & 13,614 & 0,009 \\
\hline $\begin{array}{l}\text { Existencia de alcalosis } \\
\text { respiratoria }\end{array}$ & 29,317 & 9,446 & 0,009 \\
\hline Existencia de cáncer & 32,965 & 13,093 & 0,001 \\
\hline
\end{tabular}

${ }^{a}$ Este modelo reducido es equivalente al modelo final ya que la omisión del efecto no incrementa los grados de libertad.

Tabla 6. Asociación entre la fosfatemia y los niveles séricos de creatinina, alcalosis respiratoria y la existencia de cáncer de pacientes atendidos en la emergencia.

\begin{tabular}{lcccc}
\hline & Hipofosfatemia & $\begin{array}{c}\text { Normofosfatemia } \\
\mathrm{n}(\%)\end{array}$ & $\begin{array}{c}\text { Hiperfosfatemia } \\
\mathrm{n}(\%)\end{array}$ & TOTAL \\
\hline $\begin{array}{l}\text { Creatinina sérica } \\
<0,5 \mathrm{mg} / \mathrm{dl}\end{array}$ & & & $\boldsymbol{p}=\mathbf{0 , 0 3 8}$ \\
$0,7-1,1 \mathrm{mg} / \mathrm{dl}$ & $0(0,0 \%)$ & $11(91,7 \%)$ & $1(8,3 \%)$ & $12(100 \%)$ \\
$>1,1 \mathrm{mg} / \mathrm{dl}$ & $2(4,3 \%)$ & $33(70,2 \%)$ & $12(25,5 \%)$ & $47(100 \%)$ \\
TOTAL & $2(5,4 \%)$ & $17(40,9 \%)$ & $18(48,6 \%)$ & $37(100 \%)$ \\
Alcalosis Respiratoria & $4(4,2 \%)$ & $61(63,5 \%)$ & $31(32,3 \%)$ & $96(100 \%)$ \\
Ausente & & & & $\boldsymbol{p}=\mathbf{0 , 0 3 6}$ \\
Presente & $3(15,8 \%)$ & $11(57,9 \%)$ & $5(26,3 \%)$ & $19(100 \%)$ \\
TOTAL & $1(1,5 \%)$ & $42(64,6 \%)$ & $22(33,8 \%)$ & $65(100 \%)$ \\
Cáncer & $4(4,8 \%)$ & $53(63,1 \%)$ & $27(32,1 \%)$ & $84(100 \%)$ \\
Ausente & & & $\boldsymbol{p}=\mathbf{0 , 0 0 3}$ \\
Presente & $2(2,2 \%)$ & $57(64,0 \%)$ & $30(33,7 \%)$ & $90(100 \%)$ \\
TOTAL & $2(28,6 \%)$ & $4(57,1 \%)$ & $1(14,3 \%)$ & $7(100 \%)$ \\
\hline
\end{tabular}


Tabla 7. Asociación entre el magnesio sérico y los niveles séricos de creatinina y urea.

\begin{tabular}{lcccc}
\hline & $\begin{array}{c}\text { Hipomagnesemia } \\
\mathrm{n}(\%)\end{array}$ & $\begin{array}{c}\text { Normomagnesemia } \\
\mathrm{n}(\%)\end{array}$ & $\begin{array}{c}\text { Hipermagnesemia } \\
\mathrm{n}(\%)\end{array}$ & TOTAL \\
\hline Creatinina sérica & & & & $p=0,001$ \\
$<0,5 \mathrm{mg} / \mathrm{dl}$ & $7(46,7 \%)$ & $8(53,3 \%)$ & $0(0,0 \%)$ & $15(100 \%)$ \\
$0,7-1,1 \mathrm{mg} / \mathrm{dl}$ & $3(6,1 \%)$ & $38(77,6 \%)$ & $8(16,3 \%)$ & $49(100 \%)$ \\
$>1,1 \mathrm{mg} / \mathrm{dl}$ & $7(16,7 \%)$ & $23(54,8 \%)$ & $12(28,6 \%)$ & $42(100 \%)$ \\
TOTAL & $17(16,0 \%)$ & $69(65,1 \%)$ & $20(18,9 \%)$ & $106(100 \%)$ \\
Urea sérica & & & & $p=0,001$ \\
$<0,20 \mathrm{mg} / \mathrm{dl}$ & $4(36,4 \%)$ & $7(63,6 \%)$ & $0(0,0 \%)$ & $11(100 \%)$ \\
$20-40 \mathrm{mg} / \mathrm{dl}$ & $4(10,0 \%)$ & $30(75,0 \%)$ & $6(15,0 \%)$ & $40(100 \%)$ \\
$41-80 \mathrm{mg} / \mathrm{dl}$ & $5(25,0 \%)$ & $15(75 \%)$ & $0(0,0 \%)$ & $20(100 \%)$ \\
$>80 \mathrm{mg} / \mathrm{dl}$ & $4(11,4 \%)$ & $17(48,6 \%)$ & $14(40 \%)$ & $35(100 \%)$ \\
TOTAL & $17(16,0 \%)$ & $69(65,1 \%)$ & $20(18,9 \%)$ & $106(100 \%)$ \\
\hline
\end{tabular}

ninguna variable estudiada. El calcio sérico mostró únicamente asociación significativa con la albúmina sérica (Tabla 4), mientras el fósforo sérico mostró una asociación estadística con la creatinina sérica, presencia o no de alcalosis metabólica y existencia o no de cáncer (Tablas 5 y 6). Finalmente, el magnesio sérico mostró un modelo final de regresión logística multinomial relacionada a los niveles séricos de la urea y la creatinina (Tabla 7).

\section{DISCUSIÓN}

Este estudio estuvo orientado a reconocer la frecuencia y características de los trastornos de los electrolitos, tanto mono como divalentes, de pacientes de la comunidad que acuden por diversas situaciones clínicas a la emergencia de un hospital general de Lima, Perú. Ello permite percibir la situación del medio interno del paciente sin la intervención médica hospitalaria, situación relevante para orientar un adecuado e integral enfoque clínico, indispensable para el proceso terapéutico y pronóstico del enfermo. No encontramos en la literatura trabajos equivalentes en nuestro país y son escasos en las referencias internacionales. Los existentes, hacen referencia a disturbios particulares de uno u otro componente del medio interno en particular.

El estudio fue realizado en un tamaño de muestra confiable, reconociendo que el modelo de recolección de la muestra fue accidental y ello podría constituir un sesgo, aceptable, pero que a nuestro criterio no invalidan los resultados. Este trabajo muestra una frecuencia variable de desórdenes hidroelectrolíticos de los iones monovalentes y divalentes de pacientes que acuden a nuestra emergencia, es decir tal cual provienen de su comunidad. Probablemente por ello puede diferir de estudios parecidos de la literatura (Turquía), donde la hiponatremia y la hipocalcemia fueron referidos como los trastornos más frecuentes (60 y 49\%) (8) y la hipercalcemia y la hipermagnesemia los menos frecuentes (4 y $1 \%$ respectivamente) de los pacientes atendidos en general en emergencia (8).

No encontramos en la literatura peruana información que relacione todos ellos y que permita establecer patrones de presentación que ayuden a comprender los problemas médicos en forma integral y su repercusión sobre la salud y la vida de los pacientes que acuden a la emergencia con enfermedades graves y alto riesgo de muerte. En la literatura internacional las alteraciones del fósforo son descritas como relevantes en pacientes atendidos en emergencia por una gran diversidad de sintomatología (9), y los problemas cardiovasculares y respiratorios estuvieron asociados a disturbios electrolitos en pacientes adultos mayores, mientras que solamente los cardiovasculares estuvieron asociados a los adultos menores de 65 años y los gastrointestinales a los jóvenes $(9,10)$.

La frecuencia de alteraciones encontradas en este estudio difiere de otros, incluyendo el de Meza realizado en el mismo hospital peruano, pero en pacientes ya hospitalizados varios días (11). En el gráfico 1 , vemos que la frecuencia de hiponatremia fue menor en el presente estudio igual que en el de Meza en comparación con otras referencias como los de Giordano (12) en Italia y al de Balc1 A (8) en Turquía; subrayando que la hipernatremia se observó con más frecuencia en el de Meza que en este estudio.

Los trabajos de Giordano y Ciarambino en un hospital europeo, demostraron que la variación de los niveles de sodio en un servicio de emergencia, 


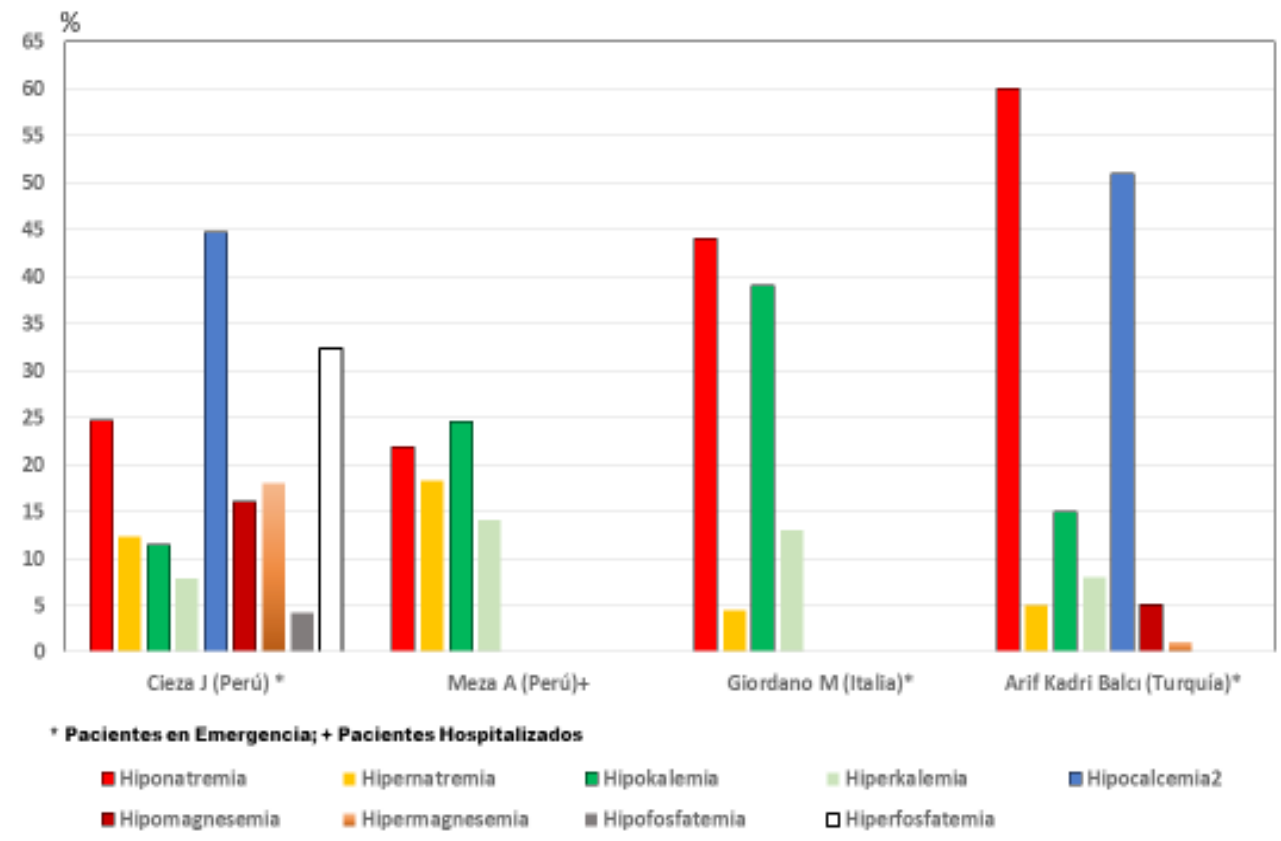

Gráfico 1. Alteraciones electrolíticas comparativas según fuentes.

estuvieron relacionados con la edad y condicionados por el ámbito estacional, explicables porque la disminución de la función renal (por efecto de la edad), aunados a la pérdida de sal, reducción de la ingesta y aumento de la ingesta de agua contribuirían al desarrollo de hiponatremia en pacientes de edad avanzada durante el verano (12). Esto es relevante porque enfatiza la dificultad en el soporte hídrico de los pacientes gravemente enfermos en la comunidad (los ancianos o pacientes postrados) o la existencia enfermedades que implican fundamentalmente pérdida de agua libre. Es también importante resaltar la elevada frecuencia de la hipocalcemia observadas en nuestro trabajo y en el de Turquía, situaciones probablemente relacionadas a desnutrición severa en estas poblaciones.

En relación al estudio peruano realizado en pacientes ya internados por más de 48 horas por Meza et al (11), debe resaltarse que, excepto una discreta disminución de la hiponatremia, se puede observar un incremento en la frecuencia de la hipernatremia y los trastornos del potasio (gráfico 1), situaciones que probablemente se originan dentro del manejo hospitalario de los pacientes y que deberían ser analizados con profundidad.

Hay pues dos hallazgos importantes. El primero es la diferencia de frecuencias de los trastornos observados en este estudio en comparación con series de países diferentes al nuestro y que pueden tener como elementos estructurales la característica de los pacientes atendidos (sobre todo el estado de nutrición), las enfermedades que los afectan y lo oportuno de la solicitud de servicio médico de emergencia. Un segundo hallazgo que se debe resaltar es la diferencia en la frecuencia de las alteraciones observadas entre los pacientes que ya están internados y los trastornos electrolíticos de los pacientes tal cual acuden a la emergencia. Estas diferencias casi con plena seguridad reflejan los efectos de la intervención médica, la misma que a la luz de los resultados, conviene se analice con objetividad y profundidad.

Sobre este último punto hay tres alteraciones que a nuestro criterio son los más importantes: el incremento de la hipernatremia, de la hiperkalemia y de la hipokalemia en pacientes hospitalizados (11), en relación a los recién admitidos a la emergencia. Si bien la hipernatremia es un fenómeno frecuente en pacientes recién admitidos en las otras dos series extranjeras mencionadas $(8,10)$, es importante subrayar el aumento de este trastorno en pacientes peruanos ya internados, que sugiere una dificultad para corregir este problema, sea por las características propias de este tipo de enfermos, o por una subestimación del problema por parte del médico, dado que la literatura lo considera un fenómeno poco frecuente como se aprecia en el gráfico 1.

Respecto al potasio, solo se encontró correlación lineal con la fosfatemia. Siendo ambos iones fundamentalmente intracelulares, es claro que ambos están ligados a la masa celular existente. Además, visto 
que la fosfatemia está relacionada a la creatinina sérica, la función renal parece jugar un rol preponderante, que si bien es cierto es estadísticamente importante en la fosfatemia, no es apreciada para el potasio. Esto probablemente ocurre por la presencia de variables confusoras como la alcalosis respiratoria propia de los estados sépticos de los pacientes aquí estudiados.

Las series revisadas describen hipopotasemia e hiperpotasemia con frecuencia de $36 \%$ y $16 \%$ respectivamente, mientras que nuestra observación encontró solamente $11,4 \%$ de pacientes con hipopotasemia y $7,8 \%$ con hiperpotasemia. No tenemos una clara explicación por la que nuestros pacientes tienen menos hipopotasemia. Álvarez et al (13), proponen a la desnutrición como factor importante de hipopotasemia, ya que en una primera instancia, en el malnutrido hay tendencia a la sobrecarga de líquidos, lo cual podría explicar la hipopotasemia. Otro factor importante de este desorden electrolítico podría corresponder a la enfermedad diarreica aguda (EDA) que según Ordóñez, es la novena causa de hospitalización (14), pero nuestro estudio no se ajusta a estas hipótesis. La baja frecuencia de hiperpotasemia sí es coherente con el pobre estado de nutrición de nuestros pacientes que acuden a las emergencias.

Respecto al calcio, el presente trabajo encontró una frecuencia relativamente alta de hipocalcemia $(46,7 \%)$, similar a lo encontrado por Balc1 A et al (8) en Turquía (51\%), pero difiere de lo hallado por Najeeb y col (15) en la India. La clara asociación de la hipocalcemia con hipoalbuminemia estaría reflejando fundamentalmente el efecto de la desnutrición traducida en la albúmina sérica (16), y sería conveniente tipificar esta variable mediante la medida del calcio iónico, situación que podría mostrar porcentajes de hipocalcemia menos frecuentes, como lo observado por otros autores (12). También es relevante mencionar que las múltiples actividades celulares en las cuales el calcio participa de una u otra forma, exige un estricto control de la concentración intracelular de este ion, recordando que a través de la membrana celular, el gradiente electroquímico para el calcio está usualmente a favor del influjo de éste hacia la célula, atravesando la membrana por canales iónicos con diferentes grados de selectividad o mediante transportadores de membrana que pueden moverlo pasiva o activamente, solo o en intercambio con otro ion, pudiendo desencadenar en ciertas situaciones apoptosis celular $(17,18)$.

A su vez, el citoplasma tiene estructuras capaces de almacenar calcio de manera activa, como las cisternas del retículo endoplasmático, de esta forma las células poseen un almacén intracelular de calcio del cual puede disponer para su actividad. Por ejemplo, del retículo sarcoplasmático de la célula muscular sale calcio cuando ellas son excitadas, y esto es esencial en el desencadenamiento del proceso contráctil (19). De otro lado, el aumento intracelular de calcio puede ser tamponado por proteínas fijadoras de éste, como la calmodulina, la calsecuestrina o la troponina. Estas proteínas, funcionan como sensores de la concentración intracelular de calcio y contribuyen a desencadenar mecanismos compensatorios que restablecen la normalidad. Finalmente, el movimiento de calcio mediado por proteínas transportadoras de membrana con intercambio $\mathrm{Na}+-\mathrm{Ca}++$ ha cobrado importancia y ha sido descrito en diferentes tejidos y células del cuerpo como epitelios, eritrocitos, células excitables y endocrinas, aunque sobre este conocimiento del trasporte de membrana hay mucho a investigar (20).

El análisis de regresión de los diferentes electrolitos mostró correlación significativa inversa entre el sodio y el fósforo y positiva entre el sodio y calcio. Esta última correlación posiblemente ocurra por efecto de la desnutrición, dado que ambos electrolitos se encuentran relacionados a los niveles de albúmina. La hiponatremia es probable suceda por la dilución plasmática y la hipocalcemia por los niveles bajos de albúmina como ya fue comentado.

En procesos celulares como la generación de potenciales de acción en las células excitables, los movimientos iónicos han sido asociados clásicamente al paso de éstos por canales específicos, pero ahora se incluye la participación del intercambiador $\mathrm{Na}+$ $\mathrm{Ca}++$. Este contra transportador mueve tres iones $\mathrm{Na}+$ contra uno de calcio, y por tanto crea una corriente positiva dirigida hacia el compartimento a donde se mueve el $\mathrm{Na}+$. Esto genera la salida de calcio de la célula, a la vez que introduce sodio, y ese es el modo directo de acción, aunque también puede actuar en forma reversa, es decir, sacar $\mathrm{Na}+$ e introducir $\mathrm{Ca}++$ (20). Por eso, en situaciones de exceso o déficit de sodio, el gradiente eléctrico generado moverá el calcio en la misma dirección lo que explicaría entonces estas correlaciones positivas entre estos dos cationes.

La relación entre el calcio y el fósforo sigue una cinética inversa, es decir, el gradiente de calcio genera un movimiento inverso de fósforo, de allí que, habiendo una correlación positiva entre el calcio y el sodio, de forma concurrente existirá una correlación inversa con el fósforo.

La correlación entre el potasio y el fósforo es mejor entendida en función que ambos son cationes intracelulares abundantes y por ello una depleción de iones intracelulares entre otras razones por desnutrición, afectará de forma similar a ambos electrolitos.

Respecto al cloro, el anión más abundante en el líquido extracelular, entra y sale de las células 
junto con el sodio y el potasio o combinado con otros cationes mayores como el calcio. Su carga negativa le permite asociarse mayormente al sodio y así es corresponsable de mantener la osmolalidad sérica y el balance hídrico. También su capacidad de poder ser transportado con cationes mayores como el calcio es lo que podría explicar la correlación positiva y significativa entre el cloro y el calcio y por consecuencia la relación inversa con el fósforo.

Una observación que hay que destacar es la relación entre el calcio y la albúmina. La hipoalbuminemia se relaciona con calcio total bajo y un calcio libre normal, es decir existe una relación directa entre los niveles de calcio y albúmina que se ve reflejada en los resultados mostrados en este trabajo ( $\mathrm{r}$ de Pearson 0,470, $p<0,02$ ). Los resultados de la regresión multinomial son acordes con lo antes ya comentado que tanto la hiponatremia como la hipocalcemia se relacionaron significativamente con la hipoalbuminemia, situación que destaca el rol que tiene la nutrición en el adecuado mantenimiento del medio interno y sobre todo con la homeostasis del sodio y del calcio.

Finalmente, el estudio mostró que la función renal alterada, expresada como una elevación de la creatinina sérica, se relaciona directamente con la posibilidad de desarrollar hiperfosfatemia, lo cual es perfectamente entendible dado que la disminución de la filtración glomerular ocasionará la acumulación del mismo en el plasma con la hiperfosfatemia consecuente (21).

En esa misma lógica, la alcalosis respiratoria, al disminuir los niveles de calcio libre aumentará los niveles del fósforo plasmático, tal cual es apreciado en el modelo final presentado en los resultados. También, no es de extrañar que, ante la elevación de la creatinina, que refleja una caída significativa de la tasa de filtración glomerular, cationes divalentes como el magnesio sufran una elevación significativa que se ve reflejado en los resultados aquí presentados y que el cáncer, una condición de hiperproliferación celular, favorezca la producción de fósforo y su elevación en el plasma como ha sido observado en otro estudio (22).

Este estudio muestra que los disturbios de los electrolitos observados en pacientes recién admitidos a la emergencia de un hospital que atiende pacientes pobres, tienen características peculiares, explicables por el estado nutricional de estos, situación que conlleva la inferencia que los disturbios de los electrolitos observados en las emergencias, no necesariamente son extrapolables de una población a otra y cada comunidad debe ser evaluada en forma específica, puesto que algunos disturbios pueden asociarse a mayor morbilidad y letalidad.

\section{Agradecimientos:}

Al Dr. Manuel Díaz De los Santos. Jefe Departamento de Emergencia. Hospital Nacional Cayetano Heredia $(\mathrm{HNCH})$ y al Dr. Raúl Valenzuela. Jefe Servicio de Nefrología $\mathrm{HNCH}$, por las facilidades brindadas.

A las señoritas Yssel Stephanny Fernánadez González y Josefina Del Rosario Fabian Quillama por el apoyo en la recolección de datos.

\section{Declaración de financiamiento y de conflictos de intereses:}

El estudio fue financiado por los autores; declaran no tener conflictos de intereses.

\section{Contribución de autoría:}

JACZ: Generación de la idea y diseño del estudio; análisis de datos, confección del informe final y compromiso de responsabilidades. CBOJ: Diseño del estudio, recolección de datos, confección del informe final y compromiso de responsabilidades

\section{Correspondencia:}

\section{Javier Cieza}

Correo electrónico: ciezaja@hotmail.com

\section{REFERENCIAS BIBLIOGRÁFICAS}

1. Vásquez-Alva R, Amado-Tineo J, RamírezCalderón F, Velásquez-Velásquez R, Huari-Pastrana R. Sobredemanda de atención médica en el servicio de emergencia de adultos de un hospital terciario, Lima Perú. An Fac med. 2016;77(4):379-85.

2. Medina G., Muñoz C. Functional reserve in functionally independent elderly persons: a calculation of gait speed and physiological cost. Rev Bras Geriatr Gerontol. (Rio de Janeiro), 2016; 19(4):577-589.

3. Cieza Z, Casillas A, Da Fieno M. Asociación del nivel de albúmina sérica y alteraciones de los electrolitos, gases sanguíneos y compuestos nitrogenados en pacientes adultos incidentes del servicio de emergencia de un hospital general. Rev Med Hered. 2016; 27:223-229.

4. Centro Nacional de Alimentación y Nutrición, Ministerio de Salud. Estado Nutricional del Perú por etapas de vida 2012-2013: Informe Técnico. Lima: Instituto Nacional de Salud; 2015. (Citado el 15 mayo del 2018) Disponible en: http://www. ins.gob.pe/repositorioaps/0/5/jer/encu_vigi_cenan/ ENUTRICIONAL\%20EVIDA\%202012-13\%20 (CTM)\%20080515.pdf 
5. Jellinge ME, Henriksen DP, Braband M. Hypoalbuminemia is a strong predictor of 30-day all-cause mortality in acutely admitted medical patiens: a prospective, observational, cohort study. PLoS One. 2014; 9(8):e105983.

6. Segura V, Régulo A, Ruiz M. Factores de riesgo de las enfermedades cardiovasculares en el Perú II. Estudio TORNASOL II comparado con TORNASOL I después de cinco años. Revista Peruana de Cardiología. 2013; 39(1):5-59.

7. Colette S, Flynn A. Sodium-calcium interrelationships with specific reference to osteoporosis. Nutrition research reviews. 1990; 3:101-115. DOI: https://doi.org/10.1079/NRR19900008

8. Balc1 A, Koksal O, Kose A, et al. General characteristics of patients with electrolyte imbalance admitted to emergency department. World J Emerg Med. 2013; 4(2):113-116.

9. Shiber JR, Mattu A. Serum phosphate abnormalities in the emergency department. J Emerg Med. 2002; 23:395-400.

10. Giordano M, Ciarambino T. Diseases associated with electrolyte imbalance in the ED: age-related differences. Am J Emerg Med. 2016;34(10):19231926.

11. Meza A, Cieza J. Frecuencia y características de las alteraciones electrolíticas en pacientes hospitalizados en servicios de Medicina en un hospital general. Rev Med Hered. 2016; 27:237-242.

12. Giordano M, Ciarambino T, Castellino $\mathrm{P}$, et al. Seasonal variations of hyponatremia in the emergency department: Age-related changes. Am J Emerg Med. 2017;35(5):749-752.doi:10.1016/j.ajem.2017.01.018

13. Alvarez A. Vasquez D. Mortalidad en pacientes desnutridos ingresados en unidad de cuidados intensios: Factores de riesgo. Rev Cubana Pediatr. 1999; 71(4):215-21.

14. Ordoñez L. Situación epidemiológica de las Enfermedades Diarreicas Agudas (EDA) en el Perú, SE 9. Boletín Epidemiológico. 2016; 25(9):152-154.
15. Najeeb Q, Ruqaya A, Sajad H, Sabiya M, Ruhi A. Electrolyte abnormalities in patients admitted in emergency department of tertiary care institute: a cross sectional study. Int J Med Sci Public Health. 2014; 3(11):1368-71.

16. Balderrama G, Suarez E. Alteraciones del calcio en niños con desnutrición proteica energética grave en el Hospital del Niño Manuel Ascensio Villarroel. Gac Med Bol. 2008; 31(1):14-17.

17. Pozzan T, Rizzuto R, Volpe P, Meldolesi J. Molecular and cellular physiology of intracellular calcium stores. Physiol Rev. 1994; 74:595-636.

18. Diaz O, Kamagate A, Herchuelz A, Van Eylen F. $\mathrm{Na} / \mathrm{Ca}$ exchanger overexpression induces endoplasmic reticulum-related apoptosis and caspase-12 activation in insulin-releasing BRIN-BD11 cells. Diabetes. 2002; 51:1815-24.

19. Fabiato A. Stimulated calcium current can both cause calcium loading in and trigger calcium release from the sarcoplsmic reticulum of a skinned canine cardiac purkinje cell. J Gen Physiol. 1985; 85:291-320.

20. Eisner DA, Lederer WJ. Na-Ca exchange: stoichiometry and electrogenicity. Am J Physiol. 1985; 248:C189-C202.

21. Sharon M. Disorders involving calcium, phosphorus, and magnesium. Prim Care. 2008; 35(2):215-216. doi: 10.1016/j.pop.2008.01.007

22. Shaikh AJ1, Bawany SA, Masood N, et al. Incidence and impact of baseline electrolyte abnormalities in patients admitted with chemotherapy induced febrile Neutropenia. J Cancer. 2011; 2:62-6.

Recibido: 05/05/2018

Aceptado: 02/07/2018 